\title{
Proton Transfer in Heterocycle Crystals
}

\author{
Iannuzzi, M ; Parrinello, M
}

\begin{abstract}
We study the proton diffusion process in imidazole-based molecular crystals, which are new candidate materials for fuel cell membranes. These materials are characterized by hydrogen bonded networks of molecules, which provide viable routes for the long-range diffusion of protons. By the application of a recently developed, powerful technique to determine reaction pathways in complex systems, we are able to reproduce the diffusion process in the imidazole crystal and in the more complicated and rigid structure of imidazole 2-ethyleneoxide. Our results cast new light on the atomistic details of the molecular rearrangements sustaining the ionic diffusion.
\end{abstract}

DOI: https://doi.org/10.1103/PhysRevLett.93.025901

Posted at the Zurich Open Repository and Archive, University of Zurich ZORA URL: https://doi.org/10.5167/uzh-138231

Journal Article

Published Version

Originally published at:

Iannuzzi, M; Parrinello, M (2004). Proton Transfer in Heterocycle Crystals. Physical Review Letters, 93(2):025901.

DOI: https://doi.org/10.1103/PhysRevLett.93.025901 


\title{
Proton Transfer in Heterocycle Crystals
}

\author{
M. Iannuzzi and M. Parrinello \\ Computational Science, Department of Chemistry and Applied Biosciences, ETH Zurich, \\ USI Campus, via G. Buffi 13, CH-6900 Lugano, Switzerland
}

(Received 9 March 2004; published 9 July 2004)

\begin{abstract}
We study the proton diffusion process in imidazole-based molecular crystals, which are new candidate materials for fuel cell membranes. These materials are characterized by hydrogen bonded networks of molecules, which provide viable routes for the long-range diffusion of protons. By the application of a recently developed, powerful technique to determine reaction pathways in complex systems, we are able to reproduce the diffusion process in the imidazole crystal and in the more complicated and rigid structure of imidazole 2-ethyleneoxide. Our results cast new light on the atomistic details of the molecular rearrangements sustaining the ionic diffusion.
\end{abstract}

DOI: 10.1103/PhysRevLett.93.025901

PACS numbers: 66.30.Hs, 71.15.Pd, 82.30.Rs

In this work we present a Car-Parrinello molecular dynamics (CPMD) study of proton diffusion in imidazole-based materials, which are promising candidate membranes in the next generation of fuel cells. Kreuer et al. [1] have demonstrated that in the condensed phase the imidazole molecules (Imi) form hydrogen bonded networks, where protons diffuse at a pace similar to that of protons in water. Thanks to the higher melting point, these materials have a competitive advantage over the more commonly used hydrated polymers and could be employed to design fuel cells operating at intermediate temperatures $\left(120-200^{\circ} \mathrm{C}\right)$. The prototype material is the imidazole crystal [2], which is formed by linear chains of hydrogen bonded molecules. Along the chains the protons are expected to move with a Grotthus-like mechanism, where the sequence of proton transfers is sustained by molecular rearrangements. The use of heterocycles as proton solvents in membranes requires their immobilization, but in such a way as to guarantee the mobility of the protons. To this end, Schuster et al. [3] have engineered a new class of materials, where the Imi chains are linked by polymeric backbones. The added rigidity increases the melting temperature. On the other hand, the question arises of how the proton diffuses, since the immobilization of the heterocycles can hinder the molecular rearrangements required for the structural diffusion process. From the influence of structural factors, such as molecular weight, polarity, the ratio of Imi and polymeric units, and the doping level, one can obtain some indications about the diffusion mechanism [4].

High resolution NMR spectroscopy [5] has shown that, even below the melting point, the proton doped samples contain locally disordered domains with reduced dipolar interactions. These domains are believed to be associated with the local rearrangement of the hydrogen bonds (HBs), which, in turn, is related to the molecular motion and the proton diffusion. The fast time scale of the rearrangement of the hydrogen bonds' patterns has prevented the determination of the microscopic diffusion mechanism [5]. However, an understanding of the diffusion mechanism is essential not only because of the relevance of proton conduction in very many areas of science, but also for a rational design of proton conductors with improved properties. For this reason, we have undertaken $a b$ initio simulations of imidazole and of imidazole 2-ethyleneoxide (Imi2eo), where the Imi molecules are tethered by a 2-ethyleneoxide spacer. We are thus able to unveil the sequence of steps characterizing the $\mathrm{H}$ diffusion in imidazole and to contrast it with the more complex process in Imi2eo.

In order to move, the proton has to clear large energy barriers, which leads to a rather slow diffusion process that cannot be observed during the short span of an $a b$ initio MD run. For this reason, we use the recently developed metadynamics (MTD) technique [6,7], which allows large barriers to be overcome in an affordable simulation time (a few picoseconds). The MTD is based on a coarse-grained, non-Markovian approach and has proven to be useful in overcoming large energy barriers and reconstructing the associated free energy profile. The main assumption is that the reaction pathway can be described as a combination of a manifold of collective variables $(\mathrm{CV})\left\{S_{\alpha}\left(\left\{\mathbf{R}_{\mathbf{I}}\right\}\right)\right\}$, which are continuous functions of the ionic coordinates $(\mathbf{R})$. These variables are more difficult to sample because they are related to the slow processes, and so they are treated separately from the other faster degrees of freedom. We associate with each selected $S_{\alpha}$ an additional variable $s_{\alpha}$ and define the dynamics of the new set of variables $\mathbf{s}$ in the space of the $\mathrm{CV}$, by the integration of the equations of motion derived from the extended Lagrangian:

$$
\begin{aligned}
\mathcal{L}= & \mathcal{L}_{\mathrm{CP}}+\sum_{\alpha} \frac{1}{2} M_{\alpha} \dot{s}_{\alpha}^{2}-\sum_{\alpha} \frac{1}{2} k_{\alpha}\left[S_{\alpha}\left(R_{I}\right)-s_{\alpha}\right]^{2} \\
& +V(t, \mathbf{s}) .
\end{aligned}
$$

In this application, $\mathcal{L}_{\mathrm{CP}}$ is the Car-Parrinello Lagrangian $[8,9]$. A fictitious mass $M_{\alpha}$ and a force constant $k_{\alpha}$, for the harmonic coupling potential, are assigned to each $s_{\alpha}$. The 
key ingredient of the method is the time-dependent potential $V(t, \mathbf{s})$, which is constructed by the accumulation of Gaussian-like hills, located in the already visited regions of the $\mathrm{CV}$ space. This potential discourages the system from remaining in the visited regions and pushes it over the energy barriers into new equilibrium basins. In order to construct a lowest energy pathway, a slow dynamics of the $\mathrm{CV}$ is recommendable, whereby the microscopic system can equilibrate at each new macroscopic configuration, and the free energy surface can be accurately sampled. It has been shown in Ref. [7] that by an appropriate choice of $M_{\alpha}$ and $k_{\alpha}$, such that the CV dynamics is much slower than the other microscopic degrees of freedom, $-V(t, \mathbf{s})$ gives the free energy dependence on $\left\{S_{\alpha}\right\}$, apart from an immaterial constant. Tests on model systems have shown [7] that the accuracy on the resolution of the free energy is given by the height of the hills that contribute to $-V(t, \mathbf{s})$.

We first study the mobility of one additional proton in the Imi crystal, which is formed by linear, hydrogen bonded chains of molecules oriented along the $c$ axis [2]. We use a simulation box containing $(2 \times 1 \times 3)$ unit cells, i.e., four parallel chains with six molecules each, and periodic boundary conditions (PBC). The proton is initially positioned between molecules II and III (see Fig. 1, state $A$ ), along the connecting HB. Upon relaxation by standard MD at $180 \mathrm{~K}$ first and by simulated annealing afterwards, the repulsion between the two close $\mathrm{H}$ atoms induces the rotation of molecule II ( $R$ defect). The charge defect $(\mathrm{Ch})$, on the other hand, migrates from molecule III to $\mathrm{V}$ via a sequence of intermolecular proton transfers. As a result, a partial inversion defect is created along the chain and, given the PBC, the $R$ and Ch defects stay as far away as possible. A farther separation between $\mathrm{Ch}$ and $R$ is observed for larger cells, which corresponds to a slight gain in energy, still negligible if compared to the activation energy required for the structural diffusion. From state $A$ (Fig. 1) we start the MTD run, with $5 \mathrm{CV}$ : the average displacement of the $\mathrm{H}$ atoms projected on the axis of the Imi chain [10] ( $k=0.5$ a.u., $M=30$ a.u.) and four angles, each taken as the angle between the normal of the plane of one Imi (II, III, IV, and V) and the chain axis ( $k=0.35$ a.u., $M=30$ a.u.) [11]. Thanks to the MTD procedure, the system is able to cross several barriers of the order of $10-15 \mathrm{kcal} / \mathrm{mol}$, which separate the intermediate states characterizing the diffusion path of the proton along the Imi chain. Most of the intermediates have similar energies and can be distinguished from the relative position of the two molecules participating in the $R$ defect. However, all the rest of the crystal is weakly affected by the specific geometry of the $R$ defect. Figure 1 shows the equilibrated structures of some of the intermediates encountered on the MTD trajectory. In less than $15 \mathrm{ps}$ the $R$ defect moves over three molecules, from II/III to V/VI. The intermediate corresponding to the lowest energy is $D$; it is a few $\mathrm{kcal} / \mathrm{mol}$ lower than $A$. In $D$, the distortion of the chain is less

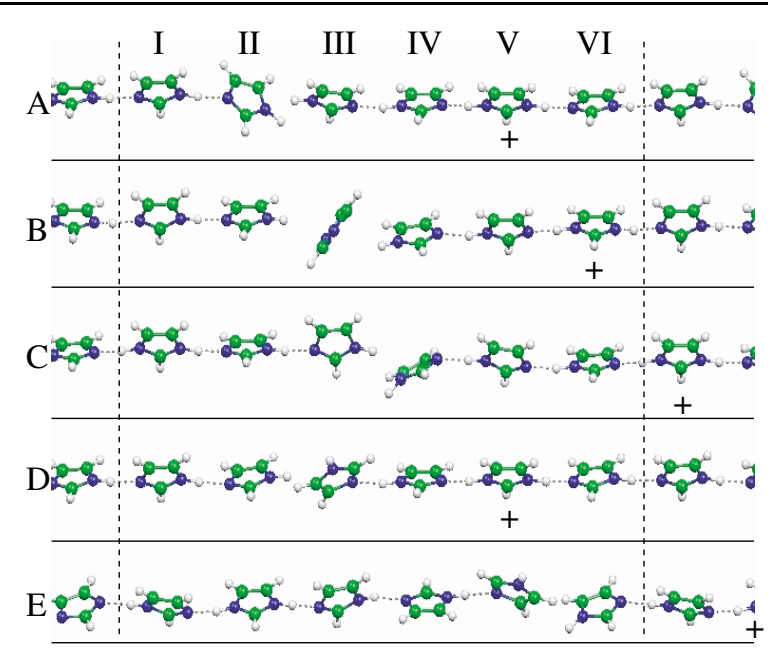

FIG. 1 (color). Equilibrated structures of five intermediates. Only the molecules in the doped chain are shown. I to VI are in the box; the others are in the periodic replicas. The + indicates the charge. $(A)$ The additional $\mathrm{H}^{+}$is on III, but the charge is on V. (B) At 2 ps, the HB between III and IV is broken; III rotates and is almost orthogonal to the chain. The charge is on VI, while the N-H bonds of II and IV are aligned and point to the center of III. $(C)$ At $3.5 \mathrm{ps,}$, the HB between II and III is restored, and the $R$ defect is in the III/IV position (similar to $A$ ). (D) At $5 \mathrm{ps}$, the $\mathrm{N}-\mathrm{H}$ bond of III is orthogonal to the chain, due to the in-plane rotation of III, while the N-H of II points towards the C-C bond of III. (E) At 8 ps, the $R$ defect is in the V/ VI position and the charge is two molecules farther.

pronounced and all the molecules lie flat along the axis. The two N-H bonds, on molecules II and III, respectively, are almost orthogonal to each other, which reduces the repulsive interaction between the two protons. In general, we observe that the chain structure can easily accommodate the defect, thanks to the large variety of metastable states, which lead to a somewhat rough free energy surface.

We now turn to the more practical case of Imi2eo. The crystalline structure of Imi2eo is composed of pairs of Imi2eo molecules linked to each other and to other pairs via $\mathrm{N}-\mathrm{H} \cdots \mathrm{N}$ bridges. A schematic picture of the primitive cell, which contains two pairs and 148 atoms, is shown in Fig. 7 of Ref. [5]. The imidazoles are arranged in chains; however, the presence of the spacers induces strong deviation from linearity.

In our calculations, we use a simulation box containing only one unit cell, i.e., four molecules per chain, to which we add one proton, in order to create the $\mathrm{Ch}$ defect. In analogy to what is observed in the Imi crystal, the relaxation of the charged structure produces an $R$ defect, due to the repulsion between the close protons ( $\mathrm{H} 1$ and $\mathrm{H} 2$ in Fig. 2, state $A$ ). State $A$ in Fig. 2 shows the equilibrated initial structure of the doped Imi2eo. The following panels show the equilibrated geometries of some other intermediates that have been encountered along the MTD trajectory. The four Imi molecules in the chain are labeled I-IV (I and II from the first pair and III and IV from the 


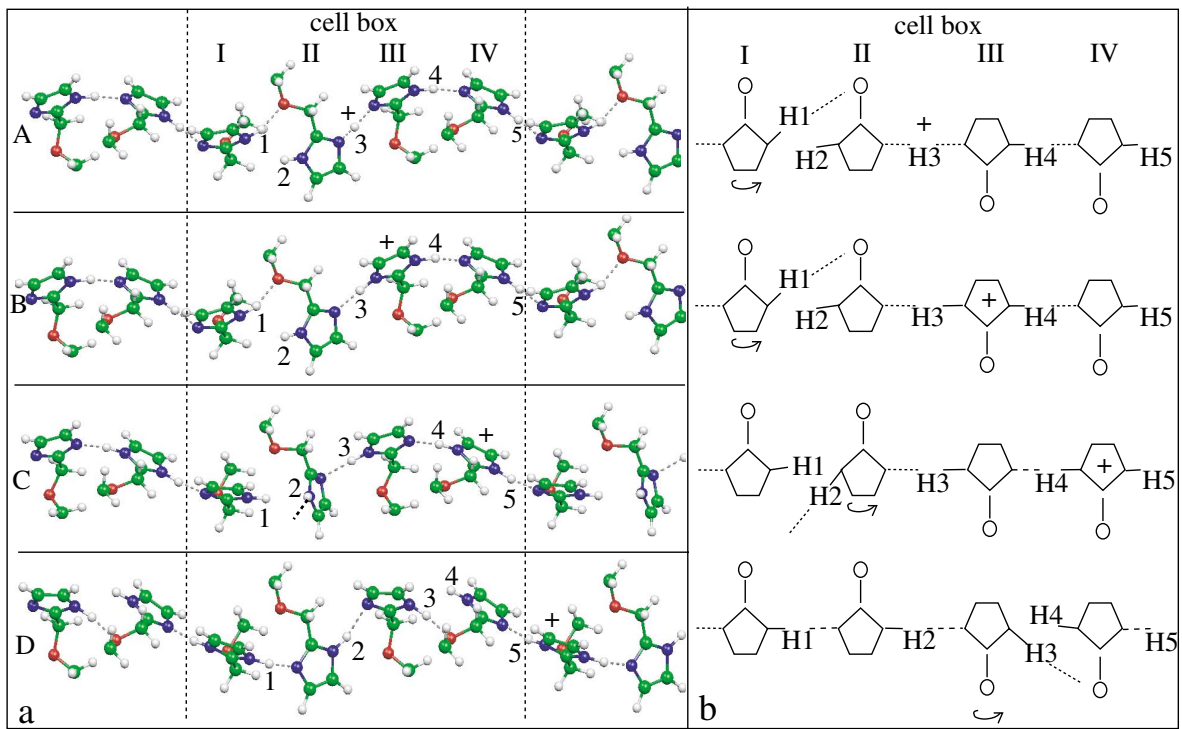

FIG. 2 (color). Optimized geometries of four intermediates of doped Imi2eo (a), and corresponding schematic representations of the HBs's pattern (b). Only the molecules in the chain and the first two segments of the spacers are shown. The relevant protons are labeled from 1 to 5 (H2: proton added in an intrapair site, $A$ ) and I to IV are the four molecules in the cell box (PBC are used). In (b) the arrow labels the molecule that is not aligned to the chain and forms the HB with one O (intra- $A B D$ or inter- $C$ pairs). ( $A$ ) First minimum; $\mathrm{H} 1$ forms a $\mathrm{HB}$ with the opposite $\mathrm{O}$ atom, and $\mathrm{H} 3$ is shared. The $\mathrm{H} \cdots \mathrm{N} H B$ on the left side of I is elongated, due to the molecular bending. (B) By MD at $200 \mathrm{~K}, \mathrm{H} 3$ moves from II to III and the $\mathrm{Ch}$ is on the III ring. (C) After 2 ps of MTD, II is orthogonal to the chain and $\mathrm{H} 2$ forms a weak $\mathrm{HB}$ with an $\mathrm{O}$ atom in a neighboring pair. The $\mathrm{N} \cdots \mathrm{H} 3 \mathrm{HB}$ is elongated due to the rotation of II and $\mathrm{Ch}$ is on IV. (D) Intrapair $R$ defect; III is rotated and forms a HB with the opposite spacer. At about 11 ps, a new state $(E)$ is found, by the rotation of IV. In $E, \mathrm{IV}$ is orthogonal to the chain and a $\mathrm{H} 4 \cdots \mathrm{O}$ is formed.

second), whereas the protons are labeled from 1 to 5 , where $\mathrm{H} 2$ is the additional proton.

In contrast to imidazole, we notice that the relaxed structure of the doped Imi2eo is characterized by the formation of one new HB, between the rotated Imi I and the closest $\mathrm{O}$ atom, in the opposite spacer of the same pair ( $A$ in Fig. 2). This is the first evidence that the complex 3D topology of Imi2eo favors the activation of additional interactions of the imidazole molecule with the atoms in the spacers, as a result of the deformation created by a defect. In the following, we show that these interactions are not restricted to the atoms of the same pair, but can occur also with atoms belonging to neighboring pairs. Moreover, we notice that the structural relaxation can be described as a local rearrangement of the pattern of the HBs, which partially compensate for the increase in energy due to the presence of the $\mathrm{Ch}$ defect and stabilize the system. The MTD shows that the $\mathrm{Ch}$ and $R$ defects diffuse together along the Imi chain through a sequence of intermediate, similar structures.

We use $5 \mathrm{CV}$ : the average displacement of the protons, projected on the Imi-chain axis (H1-H5 in Fig. 2), and four coordination numbers, one for each $\mathrm{N}$ atom sitting on the left side of the four Imi (I-IV in Fig. 2) with respect to the protons [12]. Some details about the simulation are reported in Ref. [13].

In about $15 \mathrm{ps}$, the $R$ defect moves over the whole cell through several different intermediate structures. Even if the rotational degrees of freedom of the tethered mole- cules are restricted, the protons can move from one intermolecular site to the next via the axial rotation of one Imi molecule. In fact, a complete rotation of one Imi always occurs in two or more steps, because the molecule is trapped by the formation of new HBs with the available $\mathrm{O}$ atoms on neighboring spacers. This means that the path is stepwise, and each step is characterized by a somewhat different local pattern of the HBs.

In Fig. 2, we report four typical intermediate states. In all four states, a weak $\mathrm{H} \cdots \mathrm{O}$ HB is formed between one rotated Imi and one spacer. The bond length is about $1.85 \AA$. While in the case of the $A, B$, and $D$ states the $\mathrm{O}$ atom belongs to the same pair as the imidazole molecule, in state $C$ molecule II is hydrogen bonded to an $\mathrm{O}$ in a neighboring pair (the $\mathrm{O}$ is not shown in the figure; it belongs to the pair stacked in the direction orthogonal to the chain axis). In particular, in $A, \mathrm{H} 1$ forms a weak HB with the $\mathrm{O}$ of the opposite spacer, while $\mathrm{H} 3$ is partially transferred from II to III and is shared in a Zundel-like configuration. Only a small barrier of a few $\mathrm{kcal} / \mathrm{mol}$ separates state $A$ from state $B$, which is $8 \mathrm{kcal} / \mathrm{mol}$ lower in energy. In $B, \mathrm{H} 3$ is definitively transferred to molecule III and the charge has moved to the Imi ring.

State $C$ is obtained from $B$, after a few picoseconds of MTD, when the system clears a barrier of $15 \mathrm{kcal} / \mathrm{mol}$. In this configuration, molecule II is almost orthogonal to the chain axis, and $\mathrm{H} 2$ forms a weak $\mathrm{HB}$ with an $\mathrm{O}$ atom of a neighboring pair. Since the bonding interactions characterizing $B$ and $C$ are almost equivalent, the difference in 
energy is less than $2 \mathrm{kcal} / \mathrm{mol}$. $D$, instead, is obtained through the concerted rotation of molecules II and III. In this case, two protons, $\mathrm{H} 2$ and $\mathrm{H} 3$, are transferred to the next intermolecular sites, II/III and III/IV, respectively, so the activation barrier is higher, ca. $25 \mathrm{kcal} / \mathrm{mol}$. The bonding pattern of $D$ is very similar to that characterizing $B$, but shifted to the next pair. Other shallow minima are encountered on the path from $B$ to $D$, where even weaker HBs are formed and broken, involving the $\mathrm{O}$ atoms in the closest spacers.

The MTD results demonstrate that the presence of the neighboring pairs provides additional ways to stabilize the defected structures. Namely, it is possible to activate bonding interactions when the molecule is not aligned or is even almost orthogonal to the chain axis. Further common features of the four intermediates are the presence of one $\mathrm{H}$ on the chain that does not form any $\mathrm{HB}$ and the presence of at least one elongated $\mathrm{HB}$, due to the induced bending of one Imi $(R$ defect). Even if, when the charge moves from molecule II, in $A$, to the first molecule in the right replica, in $D$, the identity of the atoms involved in the specific interactions changes, the intermediates have very similar properties. Indeed, the energies of the equilibrated structures differ only by a few $\mathrm{kcal} / \mathrm{mol}$.

Recent experimental NMR studies have revealed that upon doping new peaks appear at about $10 \mathrm{ppm}$ [5]. These peaks have been associated to unspecific "mobile" protons. This interpretation is compatible with our simulations, if we take as mobile protons those that take part in the different bonding patterns stabilizing the $R$ defects. In fact, during the simulation, the $R$ defect changes position continuously, while its bonding pattern remains very similar. Direct NMR shift calculation [14] of selected $R$ structures are in semiquantitative agreement with the experiments and show that the doping-induced peaks are to be associated with the $R$ defects. The details of these calculations will be discussed in more detail elsewhere [15].

By using MTD we are able to observe the migration of the $\mathrm{Ch}$ defect over the four molecules forming the chain, in less than 15 ps of simulation time. We show how the pattern of HBs can be locally modified due to the presence of the defect and reveal the formation of weaker HBs, which involve the atoms in the spacers. Thanks to the fast rearrangement of the hydrogen bonding, the process goes through a sequence of stable intermediate states, which are very close in energy. Actually, the interactions determining the distorted pattern of HBs are almost equivalent, even if located in different positions. The rapid change of the protons involved in these interactions explains why the measured resonances at $10 \mathrm{ppm}$, in the H-NMR spectrum, can be filtered out [16]. These interesting features characterizing the Imi-based crystals are expected to have an important role whenever the proton diffusion through a hydrogen bonded network of semi-immobilized molecules is considered.

Our method reveals the atomistic details of the molecular rearrangement responsible for the defect diffusion, as sustained by the fluctuations of the hydrogen bonding. In the case of Imi2eo, the presence of the $\mathrm{O}$ atoms in the spacers might slow down the diffusion, since they are HB acceptors that may easily trap the protons in stable, bonded configurations. On the other hand, it can decrease the activation energy for the structural diffusion, providing a stepwise diffusion mechanism, through intermediate, metastable states.

[1] K. D. Kreuer, A. Fuchs, M. Ise, M. Spaeth, and J. Maier, Electrochim. Acta 43, 1281 (1998).

[2] G. Will, Z. Kristallogr., Kristallgeom., Kristallphys., Kristallchem. 129, 211 (1969).

[3] W. H. Meyer, G. Wegner, H. G. Herz, M. Ise, M. Schuster, K. D. Kreuer, and J. Maier, Solid State Ionics 145, 85 (2001).

[4] M. F. H. Schuster, W. H. Meyer, M. Schuster, and K. D. Kreuer, Chem. Mater. 16, 329 (2004).

[5] G. R. Goward, M. H. F. Schuster, D. Sebastiani, I. Schnell, and H.W. Spiess, J. Phys. Chem. B 106, 9322 (2002).

[6] A. Laio and M. Parrinello, Proc. Natl. Acad. Sci. U.S.A. 20, 12562 (2002).

[7] M. Iannuzzi, A. Laio, and M. Parrinello, Phys. Rev. Lett. 90, 238302 (2003).

[8] R. Car and M. Parrinello, Phys. Rev. Lett. 55, 2471 (1985).

[9] CPMD V3.7 copyright IBM Corp. 1999-2001, copyright MPI für Festkörperforschung Stuttgart 1997-2001.

[10] It is a measure of the effective movement of the $\mathrm{H}$ atoms from the initial configuration in a given direction.

[11] CPMD runs are performed with Becke-Lee-Yang-Parr (BLYP) functional [A. D. Becke, Phys. Rev. A 38, 3098 (1988)], cutoff of $70 \mathrm{Ry}$, and Martins-Troullier pseudopotentials [N. Troullier and J. Martins, Phys. Rev. B 43, 1993 (1991)]. $V(t, \mathbf{s})$ is constructed by hills with height ranging between $0.6-3.8 \mathrm{kcal} / \mathrm{mol}$ and width 0.25 .

[12] The expression is given in Ref. [7]. It is ca. 1 in the $\mathrm{N}_{\text {right }} \cdots \mathrm{H}-\mathrm{N}_{\text {left }}$ configuration ( $\mathrm{N}-\mathrm{H}$ bonded), less than 1 in the $\mathrm{N}_{\text {right }}-\mathrm{H} \cdots \mathrm{N}_{\text {left }}$ configuration (HB), and it is larger than 1 when two protons share the same intermolecular site (as in $A$ and $B$ in Fig. 2).

[13] BLYP functional, 70 Ry cutoff, and Martins-Troullier pseudopotential are used. The CV mass is 5 a.u. for $s_{D}$ and 15 a.u. for the coordination numbers; $k_{\alpha}$ are 0.5 and 0.8 a.u., respectively. The hills' heights range between $0.6-5 \mathrm{kcal} / \mathrm{mol}$ and the width is 0.2 . Since the fluctuations of $s_{D}$ are larger than those of the coordination numbers, we adopt a 1.5 scaling factor for $s_{D}$ [7].

[14] D. Sebastiani and M. Parrinello, J. Phys. Chem. A 105, 1951 (2001).

[15] M. Iannuzzi and M. Parrinello (to be published).

[16] I. Schnell and H.W. Spiess, J. Magn. Reson. 151, 153 (2001). 